\title{
LA MANUFACTURA DE MUEBLES A PARTIR DE PRODUCTOS FORESTALES NO MADERABLES EN IQUITOS - PERU
}

\author{
Juan Baluarte Vásquez ${ }^{1}$
}

\section{RESUMEN}

La manufactura de muebles, a partir de productos forestales no maderables, es una actividad antigua que ocupa mano de obra y genera ingresos económicos a muchas familias de la localidad; sin embargo, no ha progresado debido a diversos factores que limitan su desarrollo. Entre los principales obstáculos que impiden el florecimiento de esta actividad, está la ausencia de mercado permanente que demande estos productos y la falta de financiamiento. Otro factor implícito es el relacionado con el escaso nivel tecnológico, una de cuyas consecuencias es la baja calidad de los productos. En Iquitos, existen entre 30 a 40 talleres familiares dedicados informalmente a esta actividad, todos estos locales no cuentan con infraestructura especializada, son de pequeño tamaño y forman parte de la vivienda del artesano. La actividad es temporal y de subsistencia, debido a los escasos ingresos económicos que genera; sin embargo, es una industria prometedora. El proceso de manufactura es totalmente artesanal, basado en operaciones tecnológicas sencillas, utilizando herramientas simples, tanto convencionales como no convencionales, y careciendo de equipo mecánico motorizado. Como resultado de este escaso nivel tecnológico, el proceso de fabricación conlleva un elevado porcentaje de desperdicios. Las principales especies que se utilizan para la manufactura de estos muebles son: tamshi (Thoracocarpus bissectus), huambé (Philodendron solimoesense), cashavara (Desmoncus sp.) y uña de gato (Uncaria guianensis, U. tomentosa).

Palabras clave: Transformación, mueblería, productos forestales no maderables, Amazonía Peruana.

1 Instituto de Investigaciones de la Amazonía Peruana. Avda. A. Quiñones km 2,5. Iquitos (Perú). E-mail: jbaluarte@iiap.org.pe 


\begin{abstract}
Furniture manufacturing from non timber forest products is a traditional activity involving labor and economic benefits to a number of local families. However, due to several development limiting factors, no substantial progress has been yet attained. The main drawbacks for this activity flourishing are the lack of a permanent market demanding these products and financial support. The low technologic level leading to low quality products is among the other factors. 30 to 40 family informal workplaces exist around Iquitos city, with no adequate infrastructure, small size and attached to the house of the artisan. This activity is temporary and of subsistence due to the low incomes generated from it, nevertheless it is a promising industry. The manufacturing process is entirely handcrafted based on an easy technology where simple conventional and non conventional tools are used, requiring no engine-operated mechanical equipment. As a consequence of this low technology the manufacturing process results in a high waste generation. The main forest species for non timber products used to make furniture are "tamshi" (Thoracocarpus bissectus), "huambe" (Philodendron solimoesense), "cashavara" (Desmoncus sp.), and "uña de gato" (Uncaria guianensis, U. tomentosa).
\end{abstract}

Key words: Manufacture, furniture, non timber forest products, Peruvian Amazon.

\title{
1. INTRODUCCION
}

En la Agenda 21 y en los Principios Forestales de la Comisión de las Naciones Unidas para el Medio Ambiente y Desarrollo -CNUMAD- (1992a, 1992b), se ha identificado a los productos forestales no maderables como un área importante que requiere de una acción concertada con el fin de asegurar su potencial para contribuir al desarrollo económico y a la generación de empleo e ingresos, de manera sostenible.

En Iquitos, se desarrolla todo un conjunto de actividades en torno a los productos forestales no maderables, en el que destaca la manufactura de muebles.

El estudio de pequeñas empresas de elaboración de productos del bosque ha sido ampliamente documentado por FAO (1990) para realidades diferentes a la nuestra. En nuestra región este tipo de actividad ha sido escasamente tratado. La fabricación de muebles, a partir de productos forestales no maderables, es una actividad que, en forma paralela a la industria formal del mueble, se viene desenvolviendo desde tiem- 
pos pretéritos en nuestra localidad; sin embargo, no ha progresado debido a diversos factores que limitan su desarrollo.

Entre los principales obstáculos, que impiden el desarrollo de esta actividad, está la ausencia de un mercado permanente que demande estos productos y la falta de financiamiento. Otro factor implícito es el relacionado con el escaso nivel tecnológico que trae la baja calidad de los productos.

Este trabajo se ha realizado con el objeto de determinar el estado de la industria del mueble, a partir de productos forestales no maderables, en Iquitos; identificar sus factores limitantes así como las posibilidades de desarrollo.

\section{METODOLOGIA}

\subsection{Area de estudio}

El estudio se realizó en la ciudad de Iquitos y en los poblados aledaños, comprendidos en el cuadrante ubicado entre las coordenadas 72 53'00" O, 3 22' 00" S y 73 21' 00" O, 04 05' 00" S. El área, pertenece a la provincia de Maynas, circunscripción de la región Loreto.

\subsection{Ejecución del estudio}

\section{Etapa de gabinete}

Está se dividió en dos sub-etapas: la primera, previa a la salida al campo, consistió en la preparación de los formularios de encuesta y la segunda, al regreso del campo, en sistematización, análisis e interpretación de resultados; en esta última etapa se utilizó el programa STAT-PAC para el procesamiento de los resultados.

\section{Etapa de campo}

Consistió en la aplicación de encuestas a 36 artesanos que se dedican a la manufactura de muebles a partir de productos forestales no maderables. Se trató, en lo posible, de cubrir el 100\% de la población artesanal; sin embargo, no se descarta la posibilidad de que se haya omitido a algunos artesanos, debido a las dificultades para localizarlos. 
Con el objeto de cubrir el mayor número posible de encuestados, se amplió la población hasta las localidades de Indiana y Tamshiyacu, ambas ubicadas en el área de influencia de Iquitos.

\section{RESULTADOS}

\subsection{Materia prima}

\section{Especies que se utilizan}

Los artesanos encuestados expresan que utilizan huambé y tamshi en porcentajes de $69 \%$ y $72 \%$ respectivamente, también afirman que utilizan cashavara y uña de gato en porcentajes similares de $31 \%$. Asimismo, el $94 \%$ de los artesanos agregan que se puede utilizar otras especies. En el Cuadro 1, se presenta las especies utilizadas en la fabricación de muebles.

Cuadro 1. Especies forestales no maderables que se utilizan en la manufactura de muebles.

\begin{tabular}{|c|c|c|c|}
\hline N. COMUN & N. CIENTIFICO & FAMILIA & USOS \\
\hline Tamshi & $\begin{array}{l}\text { Thoracocarpus bissectus } \\
\text { (Vell.) Harling }\end{array}$ & Cyclanthaceae & $\begin{array}{l}\text { Respaldos y asientos de } \\
\text { muebles típicos }\end{array}$ \\
\hline Huambé & $\begin{array}{l}\text { Philodendron } \\
\text { solimoesense A. C. Smith }\end{array}$ & Araceae & $\begin{array}{l}\text { Respaldos y asientos de } \\
\text { muebles típicos }\end{array}$ \\
\hline Cashavara & Desmoncus sp. & Arecaceae & $\begin{array}{l}\text { Respaldos y asientos de } \\
\text { muebles finos de madera }\end{array}$ \\
\hline Uña de gato & $\begin{array}{l}\text { Uncaria guianensis } \\
\text { (Aublet) Gmelia } \\
\text { U. tomentosa (Willdenow } \\
\text { ex Roemer \& Schultes) } \\
\text { DC. }\end{array}$ & Rubiaceae & $\begin{array}{l}\text { Estructura y armazón de } \\
\text { muebles típicos }\end{array}$ \\
\hline
\end{tabular}

\section{Procedencia}

El 97\% de los encuestados responde que la materia prima procede de poblaciones silvestres; correspondiendo el $29 \%$ a los bosques cercanos al río Nanay y tributarios, el $21 \%$ a los bosques ribereños de la parte baja del río Amazonas, el $15 \%$ a los 
bosques aledaños a la localidad de Tamshiyacu, el 12\% al río Napo y afluentes; mientras que el $24 \%$ expresa que provienen de otros lugares de la región Loreto.

Asimismo, el 44\% de los artesanos encuestados declara que la materia prima es extraída por ellos mismos, el $11 \%$ afirma que la adquiere en el mercado de Morona Cocha, el 3\% del río Amazonas, el 3\% del río Momón y el 39\% de diferentes lugares.

\section{Condiciones en las que se utiliza}

El $37 \%$ de los encuestados indica que la materia prima se utiliza seca, un $20 \%$ la utiliza fresca; mientras que el $31 \%$ expresa que utiliza indistintamente, es decir fresca o seca; el $11 \%$ responde que la utiliza semi-seca.

\subsection{Tecnología empleada para la manufactura de muebles}

\section{Diseños}

El 64\% manifiesta que los diseños que utiliza para la fabricación de muebles son parte de su creatividad; el $17 \%$ afirma que fabrica con base a muestras proporcionadas por los clientes; mientras que el $19 \%$ sostiene que recurre a otras fuentes como revistas, periódicos, etc.

\section{Infraestructura, equipos y herramientas}

La fabricación de estos muebles se realiza en forma casera, es decir no utiliza infraestructura especializada ni equipos industriales.

El 69\% expresa que utiliza un conjunto de herramientas constituido por alicate, machete, corta uña, tijera, lesna, sierra, cepillo, navaja, formón, escofín, cuchillo, escuadra y agujones; también manifiestan que utilizan clavo, lija y tachuela. El 31\% puntualiza que, además de las herramientas antes mencionadas, utilizan también la fibrihiladora.

\section{Rendimiento de la materia prima}

El volumen de materia prima que se utiliza depende del tamaño, modelo y tipo de tejido. El $46 \%$ contesta que adquiere en pequeña cantidad $(50 \mathrm{~kg})$, el $20 \%$ responde que compra en volúmenes de 150 a $200 \mathrm{~kg}$; mientras que el $17 \%$ precisa que compra entre 50 a $100 \mathrm{~kg}$ y un porcentaje similar afirma adquirir entre 100 a $150 \mathrm{~kg}$.

El 39\% de los artesanos declara un alto porcentaje de desperdicios (40-50\%), el 31\% dice que se pierde entre $20 \%$ a $30 \%$ de materia prima, el $28 \%$ expresa un volumen de 
desperdicios entre el $10 \%$ y $20 \%$; mientras que el $3 \%$ menciona diferentes volúmenes de desperdicios.

Asimismo, manifiestan que el 59\% de la materia prima se desperdicia en la preparación del material, el 24\% lo atribuyen a la preparación y al despunte, el $9 \%$ al despunte, el $6 \%$ por el tipo de herramienta y el $3 \%$ lo imputan a las condiciones en que se encuentra el material.

\subsection{Proceso de manufactura}

El proceso de manufactura es sencillo, está caracterizado por un conjunto de operaciones manuales y de escaso nivel tecnológico.

Para el caso del tamshi, el proceso comprende las siguientes operaciones: descortezado de la liana, deshebrado de la liana en tiras; inmediatamente, las tiras se exponen al sol para el secado; luego se procede a pulirlas para obtener fibras de sección uniforme, utilizando un fibrihilador o un machete, según la habilidad del operario; el grosor de la fibra depende del artículo a producir y la arquitectura del tejido; seguidamente se clasifica las fibras en tamaños homogéneos.

Para construir la estructura del mueble, se utiliza bejucos de uña de gato o listones de madera, dependiendo del tipo de mueble a fabricar. En el caso de los bejucos, se colecta y selecciona material de diámetro aproximado a $6 \mathrm{~cm}$; luego, se descorteza y seca; seguidamente, se uniforma el diámetro en una máquina tarugueadora hasta obtener una sección de $5 \mathrm{~cm}$ de diámetro; luego se colocan las piezas en moldes ablandando la fibra del leño con agua hervida para facilitar el moldeado, permaneciendo allí hasta que se estabilice y endurezca de acuerdo al modelo de la estructura seleccionada.

Una vez concluido el armazón, se procede al tejido del mueble, aplicando pegamento al final de cada fibra para obtener mayor consistencia y asegurar la estrecha unión de las fibras del tejido.

El acabado del mueble consiste en aplicar lija sobre la superficie áspera y fibrosa del tejido, una vez concluida esta operación se procede a aplicar laca o barniz para una mejor presentación del mueble. La Figura 1, esquematiza todo este proceso. 


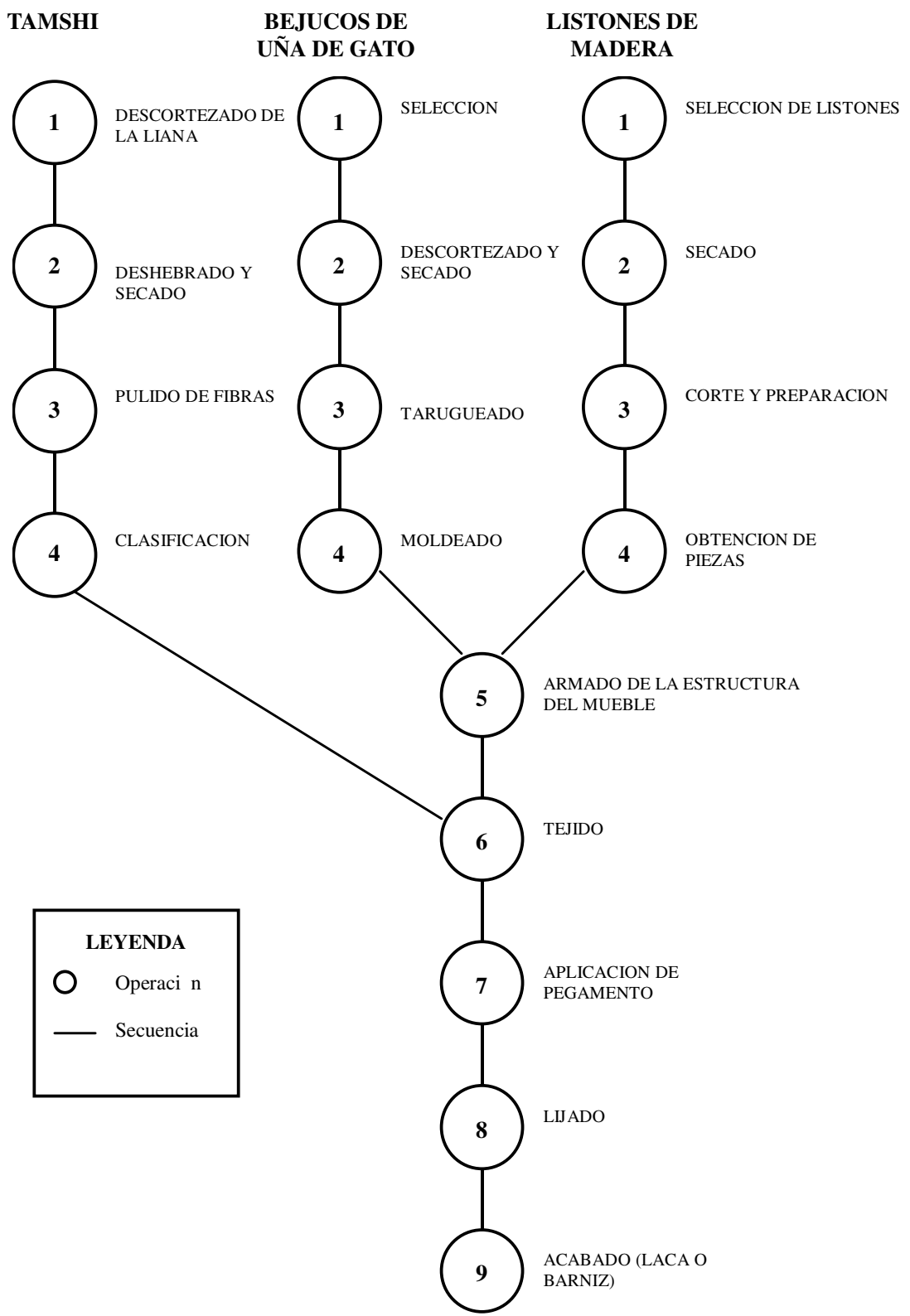

Figura 1. Proceso de manufactura de muebles a partir de cesto tamshi. 


\subsection{Comercialización}

\section{Mercado y precios}

El 75\% de los artesanos señala que la ausencia de mercado demandante, en forma permanente, es un factor limitante para la producción de estos muebles; el $11 \%$ hace constar que el mercado, junto con el financiamiento, limita el desarrollo de esta actividad; el $8 \%$ expresa que el problema está en el mercado y la materia prima en general; mientras que el 3\% opina que son el mercado, el financiamiento y la materia prima, juntos, los que limitan la actividad.

El precio por la venta de los muebles que se fabrican está en función de la oferta y la demanda en el mercado local.

\section{Flujo de comercialización}

La comercialización de estos productos es simple, generalmente se fabrican a pedido del cliente; es decir, de fabricante a cliente sin pasar por el intermediario. El cliente proporciona una cuota inicial al artesano para la adquisición de la materia prima. La cancelación del mueble es a la entrega del bien.

\subsection{Organización y tamaño de la actividad}

El 97\% de los artesanos encuestados afirma que no cuentan con licencia de funcionamiento, mientras que el 3\% expresa que posee esta documentación. Como consecuencia de esta situación, el 100\% manifiesta no pertenecer a ninguna asociación que los agrupe.

El 37\% afirma que en el taller trabaja solo una persona; el 34\% contesta que trabajan dos personas; el $11 \%$ expresa que trabajan cuatro personas; mientras que el $18 \%$ menciona que trabajan entre tres a ocho personas. Asimismo, puntualizan que la mayoría de los trabajadores son familiares, correspondiendo el $70 \%$ a hombres y el $30 \%$ a mujeres.

\subsection{Apoyo y fomento a la actividad}

Debido a la informalidad en que se desarrolla esta actividad, no cuenta con apoyo financiero de ninguna institución pública ni privada. No obstante esta situación, el 94\% de los artesanos asevera que esta actividad podría prosperar.

El 86\% de los artesanos muestra predisposición favorable hacia la capacitación, contra el $14 \%$ que afirma no estar interesado. 


\section{DISCUSIONES}

\subsection{Materia prima}

En nuestro país, la extracción, transformación y comercialización de los productos forestales no maderables está normado por el Decreto Ley 21147 «Ley Forestal y Fauna Silvestre» y por el Reglamento de Extracción y Transformación Forestal (Decreto Supremo $N^{\circ}$ 161-77-AG). Esta ley encarga al Ministerio de Agricultura la responsabilidad de normar, regular y controlar la conservación de estos recursos. Asimismo, otorga al Ministerio de Industria y Turismo, junto con el de Agricultura, la competencia de supervisar las plantas de transformación de productos del bosque.

La supervisión de este vasto sector se desarrolla sin discriminación alguna por parte de estos dos sectores públicos, dando el mismo trato tanto a las industrias de mayor envergadura como a las pequeñas. Esta situación impulsa a los artesanos dedicados a esta línea de producción a permanecer en la informalidad.

En la región Loreto, la materia prima utilizada para la transformación de productos del bosque procede mayormente del medio natural. La extracción de algunas especies tratadas en este documento ha sido reportado por Vásquez (1994) y Marmillod (1996).

Una característica particular de muchos artesanos dedicados a la manufactura de muebles, a partir de productos forestales no maderables, constituye el autoabastecimiento de la materia prima; situación que los diferencia de otras actividades afines, donde la materia prima, generalmente, es adquirida en los principales centros de expendio de nuestra localidad.

La materia prima procede de diferentes lugares de la región Loreto, la mayor parte se extrae de los bosques cercanos al río Nanay y de las quebradas principales; una cantidad considerable proviene de la parte baja del río Amazonas.

Un aspecto importante, que es necesario resaltar, es el estado en que se utiliza la materia prima, pues mientras que otros productos similares se utilizan previo secado -como el caso del mimbre (Salix viminalis), (Mansur, 1995)- un considerable número de artesanos de nuestra región la utiliza indistintamente -ya sea verde o deshidrataday otro grupo, igualmente numeroso, afirma utilizarla en estado seco. 


\subsection{Características de la actividad}

En Iquitos, existen entre 30 a 40 locales dedicados informalmente a la manufactura de muebles a partir de productos forestales no maderables, todos estos locales no cuentan con infraestructura especializada ya que forman parte de la vivienda del artesano. La actividad es prácticamente de subsistencia, debido a los escasos ingresos económicos que genera.

Utiliza pocas especies, las más utilizadas son el tamshi y el huambé, pero se utiliza también la cashavara y la uña de gato.

Los diseños que se utilizan para la fabricación de los muebles son parte de la creatividad del artesano, aunque también fabrican basándose en diseños proporcionados por los clientes, otros obtienen sus diseños de revistas, periódicos, etc.

El proceso de manufactura es totalmente artesanal, basado en operaciones tecnológicas sencillas, donde se utilizan herramientas simples tanto convencionales como no convencionales, carece de equipo mecánico motorizado.

Como producto de este escaso nivel tecnológico el proceso de fabricación deviene en un elevado porcentaje de desperdicios.

La ausencia de un mercado demandante es una seria limitante para la producción industrial de estos muebles; no obstante, consideran que el financiamiento junto con el mercado son los principales factores que limitan el despegue de esta actividad. Sin embargo, la permanencia a través del tiempo es un indicador de que existe un mercado, por lo menos local. No obstante, un factor que no fue enunciado por los encuestados pero que se evidenció en las visitas de campo es el relacionado con el escaso nivel tecnológico que trae una baja calidad de los productos.

Los artesanos dedicados a este oficio desarrollan su actividad temporal e informalmente, no integran ninguna asociación que los agrupe y, consecuentemente, no reciben apoyo financiero de ninguna entidad pública ni privada. Sin embargo, consideran que la actividad podría prosperar.

No obstante, esta actividad podría contribuir directamente al desarrollo de la mujer, éstas se encuentran relegadas, siendo su participación más notoria en la comercialización de los productos. 


\section{CONCLUSIONES}

- En el área de influencia del estudio se ha identificado 36 artesanos con igual número de establecimientos. El taller es de pequeño tamaño, de tipo familiar, no cuenta con infraestructura ni equipos especializados, se desarrolla temporal e informalmente.

- Las principales especies utilizadas para la fabricación de muebles a partir de productos forestales no maderables son: tamshi (Thoracocarpus bissectus), huambé (Philodendron solimoesense). Algunos utilizan cashavara (Desmoncus sp.) y, en pequeña cantidad, uña de gato (U. guianensis, $U$. tomentosa).

- La gran mayoría se autoabastece de materia prima, que es extraída de poblaciones silvestres procedentes de los bosques cercanos al río Nanay y tributarios, bosques ribereños al río Amazonas (parte baja), de los bosques aledaños a la localidad de Tamshiyacu y del río Napo y afluentes.

- Los diseños que se utilizan para la fabricación de los muebles son propios, también se fabrican basándose en diseños proporcionados por los clientes, otros obtienen sus diseños recurriendo a revistas, periódicos, etc.

- El proceso de fabricación es totalmente artesanal, utilizándose herramientas sencillas, tanto convencionales como no convencionales.

- $\quad$ En todo el proceso de producción se presenta un elevado porcentaje de desperdicios (20-50\%), debido fundamentalmente al escaso nivel tecnológico.

- Los principales factores que limitan el desarrollo de esta actividad son: la ausencia de un mercado permanente, la falta de financiamiento y el escaso nivel tecnológico.

- Los artesanos dedicados a esta actividad poseen escasos ingresos económicos; es decir, es una actividad de subsistencia, no están en gremios y no reciben apoyo financiero de ninguna entidad pública ni privada. 


\section{BIBLIOGRAFIA}

CONFERENCIA DE LA NACIONES UNIDAS SOBRE EL MEDIO AMBIENTE Y EL DESARROLLO. 1992a. Agenda 21. Consejo de la Tierra. Río de Janeiro, Brasil. 598 pp.

1992b. Principios Forestales. Consejo de la Tierra. Río de Janeiro, Brasil. $8 \mathrm{pp}$.

MANSUR, E. 1995. El mimbre, una pequeña industria prometedora. En: Revista Bosques y Desarrollo No 14 (38-40). Lima (Perú): Fundación Bosques y Desarrollo. Organización Internacional de Maderas Tropicales.

MARMILLOD, D. 1996. Efecto del aprovechamiento forestal sobre la población de bayal, una especie no maderable. Implicaciones ecológicas para una silvicultura con fines de producción diversificada en bosques de El Petén, Guatemala. Primer borrador, documento de trabajo. Turrialba (Costa Rica): CATIE. $11 \mathrm{pp}$.

ORGANIZACION DE LAS NACIONES UNIDAS PARA LA AGRICULTURA Y LA ALIMENTACION (FAO). 1990. Pequeñas empresas de elaboración de productos del bosque. Primera reimpresión 1993. Roma (Italia): FAO. $290 \mathrm{pp}$.

VASQUEZ, M. 1994. Extracción y comercialización de productos forestales diferentes de la madera en el ámbito de la Unidad Forestal de Iquitos - Perú. Tesis para optar el título de Ingeniero Forestal UNAP. Iquitos (Perú). $123 \mathrm{pp}$. 


\section{FOLIA AMAZÓNICA - IIAP}

ISSN: $1018-5674$

SUSCRIPCIÓN ANUAL*/ANNUAL SUSCRIPTION*

\begin{tabular}{lccc}
\hline & $\begin{array}{c}\text { NACIONAL/ } \\
\text { LOCAL }\end{array}$ & AMÉRICA & $\begin{array}{c}\text { RESTO DEL } \\
\text { MUNDO }\end{array}$ \\
\hline PERSONAL & US\$ 20 & US\$ 25 & US\$ 35 \\
INSTITUCIONAL & US\$ 30 & US\$ 35 & US\$ 45 \\
\hline
\end{tabular}

FORMA DE PAGO / PAYMENT

- Contado - Master Card - Visa - Transferencia Bancaria

- Enviar el pago a la cuenta:

Nuevos Soles S/. Bco. Interbank $=740-821776-7$

Dollars US\$ Bco. de Crédito = 390-1059267168

\section{SOLICITANTE}

- Nombre

- Dirección

- Institución :

- Dirección :

\section{INFORMES}

Área de Publicaciones

Av. Abelardo Quiñones km 2.5

Apartado Aéreo 784 Iquitos - Perú

Telf.: (00 51) 094265515 - 265516

Fax: (00 51) 094265527

E-mail: occt@iiap.org.pe • cdi@iiap.org.pe

* No cubre gastos de envío aéreo. 